\title{
SYNTHESIS OF ANTHRA[2,3-b]BENZOFURAN DERIVATIVES BY CYCLIZATION OF LACCAIC ACID A DERIVATIVES AND ITS REACTION MECHANISM
}

Dingyu $\mathrm{Hu}$, Yoshihiko Shinoda, and Shin-ichi Nakatsuka*

The United Graduate School of Agricultural Science, Gifu University, Yanagido, Gifu 501-11, Japan

Abstract: Cyclization conditions and reaction mechanism for transformation of laccaic acid A 1 to an anthra[2,3-b]-benzofuran 2 were studied preparing 3-methoxy derivatives of 1 , such as 4 6, and a new pathway for the cyclization was proposed, and several related compounds $7 \sim 9$ were synthesized.

Lac-dye is a red pigment isolated from sticklac produced by a tiny insect, Laccifer lacca, in India and Southeast Asia, and has been used for over a thousand years. Lac-dye, the watersoluble colouring matter, has been shown to be a mixture of at least five constituents (laccaic acid $A, B, C, D$ and $E$ ), and laccaic acid $A 1(1)$ is the major component. In the course of their chemical studies, two groups(1b,1c) have reported a unique cyclization reaction of laccaic acid $\mathrm{A}$ to anthra[2,3-b]benzofuran derivative 2 , which occurs during methylation of 1 with

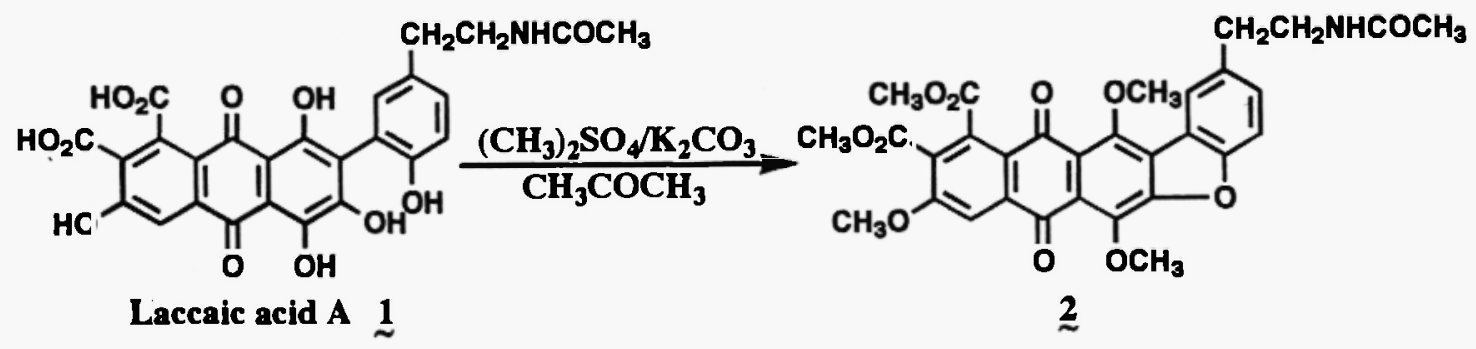

E. D. Pandhare et al., 1966

R. Wurwood et al., 1967 
$\left(\mathrm{CH}_{3}\right)_{2} \mathrm{SO}_{4}$ in the presence of potassium carbonate in boiling acetone. Their proposed reaction mechanism via (a) tautomerization in which the 1,4-hydroxyl groups and 9,10-quinone functions are interchanged, (b) methylation of a hydroxyl group at 3-position of the anthraquinone ring and (c) displacement of a 3-methoxyl group of the anthraquinone ring by the phenolate ion of the adjacent phenyl ring, is similar to the one suggested by Barton and Scott(2) in the formation of xanthone from 2-hydroxy-2'-methoxybenzophenone. We studied this cyclization reaction using 3-methoxy derivatives as substrates, and wish to report that the step (a) is not essential for the cyclization.

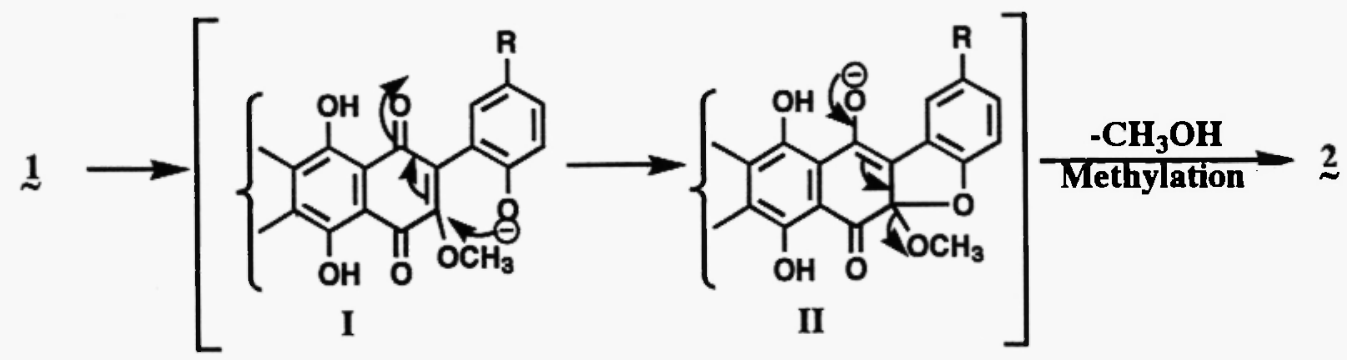

Scheme 1

Our studies started from preparation of 3-( $O$-methyl)anthraquinone derivatives of laccaic acid A. Laccaic acid A 1 was partially methylated under mild conditions $\left(\mathrm{CH}_{3} \mathrm{I}, \mathrm{K}_{2} \mathrm{CO}_{3} / \mathrm{DMF}\right.$ at $55^{\circ} \mathrm{C}$ for $12 \mathrm{~h}$ ), and the corresponding tetramethyl derivative 4 (3) and pentamethyl derivative 5(4) were isolated from the reaction mixture by a combination of silica gel column, prep.TLC and HPLC (Namsil 9, 20 $\phi \times 250 \mathrm{~mm}$ ) in $12.7 \%$ and $14.4 \%$ yields, respectively(5). Xantholaccaic acid A 3, prepared by reduction of laccaic acid A 1(1d), was also partially methylated under the same reaction conditions to afford tetramethylxantholaccaic acid 6(6) in $25 \%$ yield(5). In all of these three compounds(4, 5 and 6), two carboxyl groups at 7- and 8positions and two phenolic hydroxyl groups at 3-and 6-positions were methylated, but phenolic $\mathrm{OH}$ of a substituent at 2-position of the anthraquinone ring was not methylated.

By the treatment of the compounds 4,5 and 6 with $\mathrm{K}_{2} \mathrm{CO}_{3}$ in DMF at $60^{\circ} \mathrm{C}$ for $6 \mathrm{~h}$, anthra[2,3-b]benzof uran derivatives $7(7), 8(8)$ and $9(9)$ were obtained in good yield (74\%, $72 \%$ and $72 \%$, respectively). The cyclization rates of 4,5 and 6 were almost the same in each case even if the compounds 5 and 6 could not tautomerize to the 1,4-anthraquinone form. Furthermore, the cyclization was not observed when the hydroxyl groups at 3-position were not methylated such as in the case of 1, 3, and their dimethyl esters. Therefore, meth- 
ylation of the 3-hydroxyl group is essential for the cyclization, which proceeds via direct displacement of a methoxyl group at 3-position of 9,10-anthraquinone by the phenolate ion of the adjacent phenyl ring, as shown in Sheme 2. Thus we propose that the tautomeric isomerization is not an essential factor in the formation of anthra[2,3-b]benzof unan derivatives from laccaic acids. Further study of laccaic acids is now in progress.
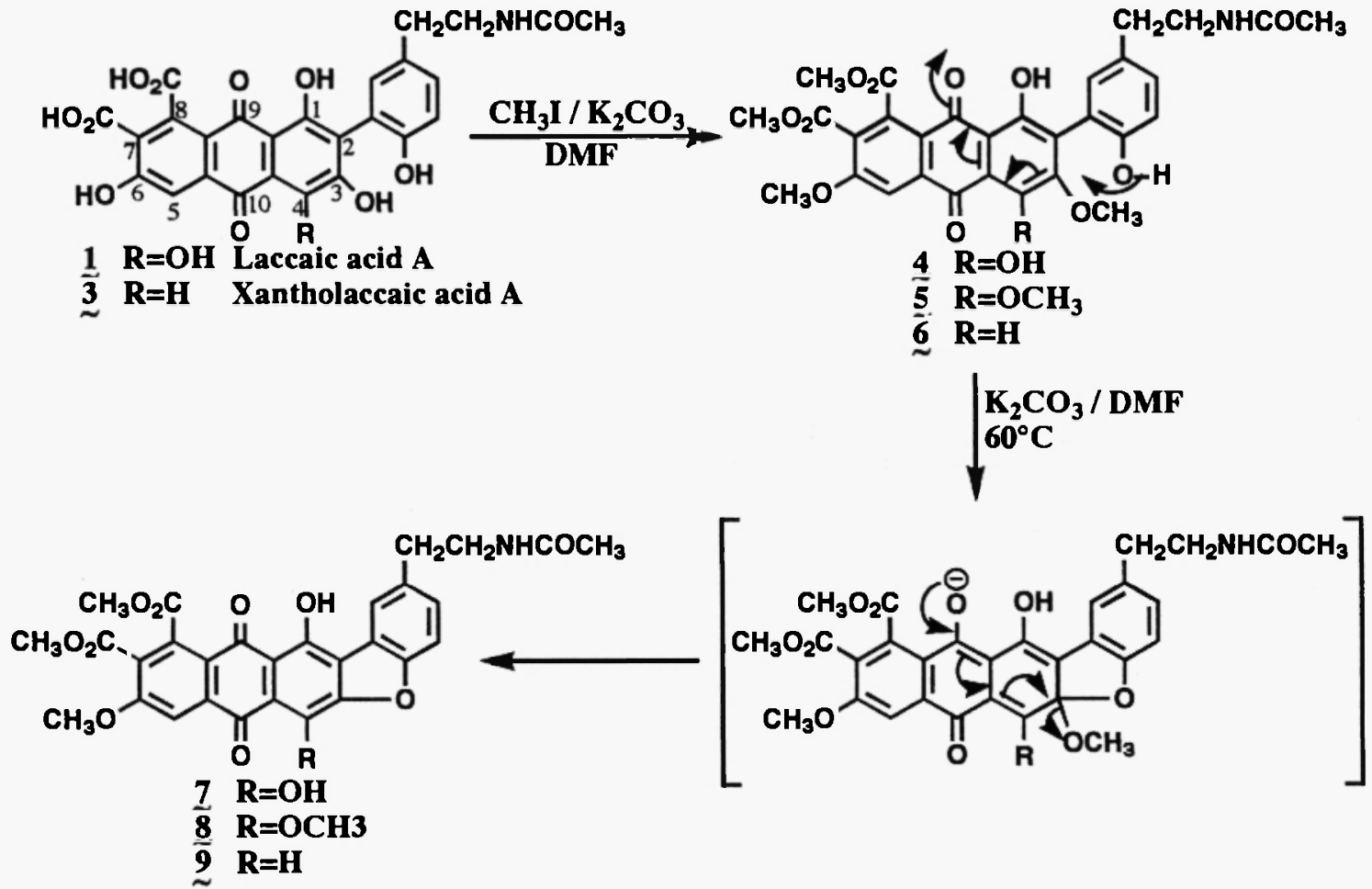

Scheme 2

\section{REFEREN CES AND NOTES}

(1) a) R. Burwood, G. Read, K. Schofield \& D. E. Wright, J. Chem. Soc., 1965, 6067, b) E. D. Pandhare, A. V. Rama Rao, R. Srinivasan \& K. Venkataraman, Tetrahedron Suppl., 8, Part 1, 229(1966); c) R. Burwood, G. Read, K. Schofield \& D. E. Wright, J. Chem. Soc., (C), 1967,842 ; d) E. D. Pandhare, A. V. Rama rao \& I. N. Shaikh, J. Chem., 7, 977(1969).

(2) a) D. H. R. Barton and A. I. Scott, J. Chem. Soc., 1958, 1767; b) W. J.McMaster, A. I. Scott and J. Tripett, J. Chem. Soc., $1960,4628$. 
(3) 4: ${ }^{1} \mathrm{H}-\mathrm{NMR}\left(\mathrm{CDCl}_{3}\right) \delta \mathrm{ppm} 1.96(3 \mathrm{H}, \mathrm{s}), 2.78(2 \mathrm{H}, \mathrm{t}, \mathrm{J}=7.0), 3.50(2 \mathrm{H}, \mathrm{m}), 3.87(3 \mathrm{H}$, s), 3.93(3H,s $), 3.95(3 \mathrm{H}, \mathrm{s}), 4.09(3 \mathrm{H}, \mathrm{s}), 7.01(1 \mathrm{H}, \mathrm{d}, \mathrm{J}=8.3), 7.08(1 \mathrm{H}, \mathrm{d}, \mathrm{J}=2.0)$, 7. 17( $1 \mathrm{H}, \mathrm{dd}, \mathrm{J}=8.3,2.0), 7.96(1 \mathrm{H}, \mathrm{s}) ; \mathrm{UV}\left(\mathrm{CH}_{3} \mathrm{OH}\right) \lambda_{\max } \mathrm{nm}(\varepsilon) 220(29,700), 241$ $(24,500), 282(25,000), 327(12,200) .496(11,400)$. IR(KBr) vcm-1 3,377, 1,743, $1,724,1,656,1,578,1,440$.

(4) 5: ${ }^{1} \mathrm{H}-\mathrm{NMR}\left(\mathrm{CDCl}_{3}\right) \delta \mathrm{ppm} 1.96(3 \mathrm{H}, \mathrm{s}), 2.80(2 \mathrm{H}, \mathrm{t}, \mathrm{J}=7.1), 3.50(2 \mathrm{H}, \mathrm{m}), 3.77(3 \mathrm{H}$, s), $3.93(3 \mathrm{H}, \mathrm{s}), 3.94(3 \mathrm{H}, \mathrm{s}), 3.99(3 \mathrm{H}, \mathrm{s}), 4.08(3 \mathrm{H}, \mathrm{s}), 7.03(1 \mathrm{H}, \mathrm{d}, \mathrm{J}=8.2), 7.09$ $(1 \mathrm{H}, \mathrm{d}, \mathrm{J}=2.0), 7.17(1 \mathrm{H}, \mathrm{dd}, \mathrm{J}=8.2,2.0), 7.86(1 \mathrm{H}, \mathrm{s}) ; \mathrm{UV}\left(\mathrm{CH}_{3} \mathrm{OH}\right) \lambda_{\max } \mathrm{nm}(\varepsilon)$ $225(34,100), 277(29,800), 441(7,400)$; IR(KBr) vcm-1 3,400, 1,730, 1,620, 1,580, $1,440,1,380$.

(5) Over ten kinds of methylated compounds were obtained from the reaction mixture and all structures were determined. The methylated positions were determined by comparis on of their ${ }^{1} \mathrm{H}$ and ${ }^{13} \mathrm{C}$ NMR spectra.

(6) 6: ${ }^{1} \mathrm{H}-\mathrm{NMR}\left(\mathrm{CDCl}_{3}\right) \delta \mathrm{ppm} 1.92(3 \mathrm{H}, \mathrm{s}), 2.85(2 \mathrm{H}, \mathrm{t}, \mathrm{J}=7.0), 3.50(2 \mathrm{H}, \mathrm{m}), 3.90(3 \mathrm{H}$, $\mathrm{s}), 3.91(3 \mathrm{H}, \mathrm{s}), 3.92(3 \mathrm{H}, \mathrm{s}), 4.05(3 \mathrm{H}, \mathrm{s}), 6.98(1 \mathrm{H}, \mathrm{d}, \mathrm{J}=8.3), 7.0(1 \mathrm{H}, \mathrm{d}, \mathrm{J}=2.1)$, 7. $11(1 \mathrm{H}, \mathrm{dd}, \mathrm{J}=8.3,2.1), 7.38(1 \mathrm{H}, \mathrm{s}), 7.74(1 \mathrm{H}, \mathrm{s}) ; \mathrm{UV}\left(\mathrm{CH}_{3} \mathrm{OH}\right) \lambda \max \mathrm{nm}(\varepsilon) 220$ $(21,200), 287(19,000), 335 \mathrm{sh}(3,500), 421(3,600) ; \mathrm{IR}\left(\mathrm{CH}_{2} \mathrm{Cl}_{2}\right) v \mathrm{~cm}^{-1} 3,400,1,745$. $1,670,1,635,1,585,1,325$.

(7) 7: ${ }^{1} \mathrm{H}-\mathrm{NMR}\left(\mathrm{CDCl}_{3}\right) \delta \mathrm{ppm} 1.95(3 \mathrm{H}, \mathrm{s}), 3.00(2 \mathrm{H}, \mathrm{t}, \mathrm{J}=7), 3.60(2 \mathrm{H}, \mathrm{m}), 3.95(3 \mathrm{H}, \mathrm{s})$, $4.03(3 \mathrm{H}, \mathrm{s}), 4.10(3 \mathrm{H}, \mathrm{s}), 7.42(1 \mathrm{H}, \mathrm{dd}, \mathrm{J}=8.2,2.1), 7.61(1 \mathrm{H}, \mathrm{d}, \mathrm{J}=8.2), 7.94$ $(1 \mathrm{H}, \mathrm{s}), 8.07(1 \mathrm{H}, \mathrm{d}, \mathrm{J}=2.1) ; \mathrm{UV}\left(\mathrm{CH}_{3} \mathrm{OH}\right) \lambda \max \mathrm{nm} 217,282,340,434 ; 1 \mathrm{R}\left(\mathrm{CH}_{2} \mathrm{Cl}_{2}\right)$ $v_{c m}^{-1} 3,313,1,738,1,655,1,578,1,431,1,344$.

(8) $8:{ }^{1} \mathrm{H}-\mathrm{NMR}\left(\mathrm{CDCl}_{3}\right) \delta \mathrm{ppm} 1.95(3 \mathrm{H}, \mathrm{s}), 2.92(2 \mathrm{H}, \mathrm{t}, \mathrm{J}=7.0), 3.57(2 \mathrm{H}, \mathrm{m}), 3.94(3 \mathrm{H}$, $\mathrm{s}), 4.02(3 \mathrm{H}, \mathrm{s}), 4.06(3 \mathrm{H}, \mathrm{s}), 4.27(3 \mathrm{H}, \mathrm{s}), 7.42(1 \mathrm{H}, \mathrm{dd}, \mathrm{J}=8.5,2.1), 7.62(1 \mathrm{H}, \mathrm{d}$, $\mathrm{J}=8.5), 7.80(1 \mathrm{H}, \mathrm{s}), 8.10(1 \mathrm{H}, \mathrm{d}, \mathrm{J}=2.1) ; \mathrm{UV}\left(\mathrm{CH}_{3} \mathrm{OH}\right) \lambda \max \mathrm{nm}(\varepsilon) 221(9,800), 281$ $(4,100), 449(3,600)$; IR(KBr) vcm-1 3,400,1,740,1,650,1,580,1,415, 1,340.

(9) 9: ${ }^{1} \mathrm{H}-\mathrm{NMR}\left(\mathrm{CDCl}_{3}\right) \delta \mathrm{ppm} 1.97(3 \mathrm{H}, \mathrm{s}), 3.00(2 \mathrm{H}, \mathrm{t}, \mathrm{J}=7.0), 3.54(2 \mathrm{H}, \mathrm{t}, \mathrm{J}=7.0)$, $3.95(3 \mathrm{H}, \mathrm{s}), 4.04(3 \mathrm{H}, \mathrm{s}), 4.10(3 \mathrm{H}, \mathrm{s}), 7.42(1 \mathrm{H}, \mathrm{dd}, \mathrm{J}=8.5,2.1), 7.85(1 \mathrm{H}, \mathrm{d}, \mathrm{J}=$ 8.5), 7.92(1H,s), 8. $10(1 \mathrm{H}, s), 8.40(1 \mathrm{H}, \mathrm{d}, \mathrm{J}=2.1) ; \mathrm{UV}\left(\mathrm{CH}_{3} \mathrm{OH}\right) \lambda_{\max } \mathrm{nm}(\varepsilon) 221$ $(32,200), 279(47,200), 340(5,000), 433(9,810) ; \operatorname{IR}\left(\mathrm{CH}_{2} \mathrm{Cl}_{2}\right) \mathrm{vcm}-11,742,1,675$, $1,640,1,585,1,340$.

Received on November 5, 1996 